\title{
Leuconostoc sp en pacientes con cáncer: Estudio descriptivo
}

\author{
Sonia I. Cuervo M., Jorge Cortés L., Elizabeth Rodríguez R., Natan Hormaza A. y Erwing Vargas S.
}

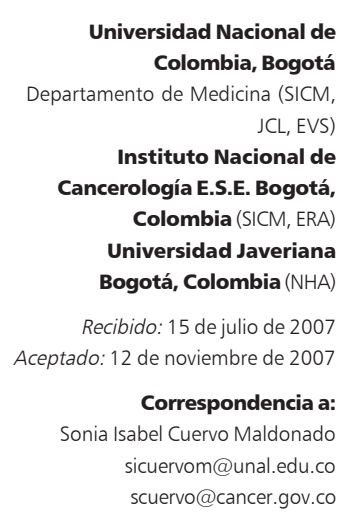

\section{Introducción}

$\mathrm{L}$ as bacterias pertenecientes al género Leuconostoc se consideran lactacidobacterias de amplio conocimiento y utilidad en la industria alimenticia por sus propiedades fermentadoras y para la generación de compuestos odoríferos. Estas cocáceas o cocobacilos grampositivos no esporulados, son microaerofílicos con grandes exigencias nutricionales para su crecimiento en medios de cultivo y pertenecen a la familia Streptococcaceae. En la naturaleza se encuentran en gran variedad de ecosistemas vegetales.

En su descripción inicial en el ámbito clínico no se consideraban patógenas; sin embargo, durante los últimos 20 años su mención en series y reportes de casos ha permitido reconocerlas como agentes patógenos oportunistas, predominantemente en pacientes inmunocomprometidos con infecciones intrahospitalarias ${ }^{1}$.

Todas las especies de Leuconostoc son resistentes a la capacidad lítica de vancomicina, debido a que los precursores pentapéptidos de la pared celular terminan en un depsipéptido (alanina-lactato) y no en el dipéptido alanina-alanina; este último es el sitio de acción para vancomicina en las cocáceas grampositivas que son sensibles a este antimicrobiano. Este microorganismo presenta, generalmente, resistencia cruzada con teicoplanina. Se ha descrito resistencia a sulfonamidas, cotrimoxazol, ácido fusidico y fosfomicina ${ }^{2}$. Los antimicrobianos a los cuales ha mostrado susceptibili- dad son: penicilina, ampicilina, clindamicina, eritromicina y gentamicina, razón por la que se consideran de primera elección cuando se presenta infección por Leuconostoc sp.

Describimos a continuación una población de pacientes con cáncer en quienes se identificó Leuconostoc sp en el contexto de infecciones nosocomiales o relacionadas con la neoplasia de base y, en la mayoría de los casos, formando parte de una flora polimicrobiana causante de la patología infecciosa ${ }^{1}$.

\section{Pacientes y Métodos}

Estudio descriptivo, retrospectivo, en el que se revisaron las historias clínicas de pacientes atendidos en el Instituto Nacional de Cancerología (INC) E.S.E en Bogotá, Colombia, con reporte de cultivo positivo para Leuconostoc sp en cualquier muestra clínica, en el período: septiembre de 2004 a julio de 2006. Los datos se recolectaron en un formato diseñado para tal fin y se utilizó una base de datos de Excel (Microsoft Office 9.0.3821).

Entre 2004 y 2006 se utilizó en el laboratorio de microbiología del INC el método automatizado MicroScan ${ }^{\circledR}$ para la identificación de microorganismos comunes y la realización de las pruebas de susceptibilidad por microdilución. Específicamente para Leuconostoc, cocácea grampositiva catalasa negativa, la sospecha del laboratorio surgió de la observación de las características del crecimiento en agar sangre enri- 
quecido con sangre de cordero, colonias con tamaño significativamente menor que las correspondientes al género Streptococcus, y de la resistencia a vancomicina por el método automatizado, confirmado por difusión en agar. Para todos los antibacterianos ensayados se utilizaron los dos métodos.

\section{Resultados}

Se identificaron nueve pacientes con neoplasias hematológicas y no hematológicas. Los principales hallazgos clínicos y microbiológicos se presentan en la Tabla 1.

Se identificaron siete mujeres y dos hombres con edades comprendidas entre siete y 76 años; cinco pacientes presentaban tumores hematológicos (tres leucemias, un linfoma no Hodgkin y un mieloma múltiple). Las neoplasias restantes correspondieron a un adenocarcinoma de colon y uno de recto, un carcinoma de cérvix y un carcinoma broncogénico.

Leuconostoc sp se identificó como único agente infeccioso en cuatro pacientes, tres con tumores hematológicos y uno con adenocarcinoma de recto. En los pacientes con tumores hematológicos, Leuconostoc sp se recuperó del torrente sanguíneo, siendo interpretado como bacteriemia primaria, durante un episodio de neutropenia febril post-quimioterapia y en una paciente se identificó en el catéter vascular central y en dos hemocultivos periféricos, lo cual confirmó el diagnóstico de bacteriemia asociada a CVC, secundaria al uso de nutrición parenteral por una patología neoplásica gastrointestinal.

En los cinco pacientes restantes, Leuconostoc sp se identificó como parte de infecciones polimicrobianas causadas por bacilos gramnegativos, otras cocáceas grampositivas y Candida albicans. En el paciente en quien se aisló de coprocultivo, en el contexto de una colitis neutropénica, la interpretación de este aislado es relevante desde el punto de vista clínico si se tiene en cuenta que el paciente cursaba con una neutropenia absoluta y grave (cero neutrófilos) y para el caso de la úlcera perianal, su detección asociada a Candida albicans, probablemente obedeció más a colonización que a una infección; sin embargo, la paciente también cursaba en el momento de la identificación con neutropenia y fiebre. La identificación en la muestra de lavado broncoalveolar en un paciente con neumonía post-obstructiva por carcinoma broncogénico escamocelular en progresión es de interpretación clínica más difícil; considerando que el informe de la muestra arrojó un recuento de $10^{4}$ colonias/ml de secreción bronquial, en un paciente francamente sintomático induce al médico clínico a considerar la posibilidad de adecuar el tratamiento antimicrobiano al resultado del laboratorio. En los dos casos en los cuales se aisló de procesos

\begin{tabular}{|c|c|c|c|c|c|c|c|}
\hline $\mathbf{n}$ & $\begin{array}{c}\text { Edad (años) } \\
\text { H/M }\end{array}$ & Neoplasia & Factor asociado & IIH & Microbiología & $\begin{array}{l}\text { Susceptibilidad } \\
\text { a penicilina }\end{array}$ & Desenlace \\
\hline 1 & $44 \mathrm{M}$ & $\begin{array}{l}\text { Carcinoma de cuello } \\
\text { uterino }\end{array}$ & $\begin{array}{l}\text { Estadio IIB en } \\
\text { progresión }\end{array}$ & $\begin{array}{l}\text { Absceso de pared } \\
\text { abdominal }\end{array}$ & $\begin{array}{l}\text { E. coli, E. faecalis y } \\
\text { Leuconostoc sp }\end{array}$ & $S$ & Muerte \\
\hline 2 & $7 \mathrm{H}$ & $\begin{array}{l}\text { Leucemia linfoblástica } \\
\text { aguda }\end{array}$ & $\begin{array}{l}\text { Neutropenia febril } \\
\text { post-quimioterapia }\end{array}$ & Bacteriemia primaria & Leuconostoc sp & $S$ & Mejoría \\
\hline 3 & $19 \mathrm{M}$ & $\begin{array}{l}\text { Leucemia linfoblástica } \\
\text { aguda }\end{array}$ & $\begin{array}{l}\text { Neutropenia febril } \\
\text { post-quimioterapia }\end{array}$ & Bacteriemia primaria & Leuconostoc sp & S & Muerte \\
\hline 4 & $78 \mathrm{M}$ & $\begin{array}{l}\text { Adenocarcinoma de } \\
\text { colon }\end{array}$ & $\begin{array}{l}\text { Intestino corto, } \\
\text { obstrucción intestinal }\end{array}$ & Peritonitis secundaria & $\begin{array}{l}\text { E. faecium, K. pneu- } \\
\text { moniae, Leuconostoc sp }\end{array}$ & $S$ & Muerte \\
\hline 5 & $43 \mathrm{M}$ & $\begin{array}{l}\text { LNH difuso de células } \\
\text { grandes }\end{array}$ & $\begin{array}{l}\text { Neutropenia febril } \\
\text { post-quimioterapia }\end{array}$ & Colitis neutropénica & S. miitis, Leuconostoc sp & 1 & Mejoría \\
\hline 6 & $50 \mathrm{M}$ & Mieloma múltiple & Preparación para TAMO & Bacteriemia primaria & Leuconostoc sp & $S$ & Mejoría \\
\hline 7 & $35 \mathrm{M}$ & $\begin{array}{l}\text { Adenocarcinoma de } \\
\text { recto }\end{array}$ & $\begin{array}{l}\text { Pseudo-obstrucción } \\
\text { intestinal }\end{array}$ & $\begin{array}{l}\text { Bacteriemia asociada } \\
\text { a CVC }\end{array}$ & Leuconostoc sp & $S$ & Mejoría \\
\hline 8 & $8 \mathrm{M}$ & $\begin{array}{l}\text { Leucemia linfoblástica } \\
\text { aguda }\end{array}$ & $\begin{array}{l}\text { Neutropenia febril } \\
\text { post-quimioterapia }\end{array}$ & Úlcera perianal & C. albicans, Leuconostoc sp & $S$ & Mejoría \\
\hline 9 & $76 \mathrm{H}$ & Carcinoma broncogénico & $\begin{array}{l}\text { Estadio IIIA en } \\
\text { progresión }\end{array}$ & $\begin{array}{l}\text { Neumonía post- } \\
\text { obstructiva }\end{array}$ & $\begin{array}{l}\text { SAMS } \\
\text { Leuconostoc sp }\end{array}$ & $S$ & Mejoría \\
\hline
\end{tabular}


infecciosos activos (absceso de la pared abdominal y peritonitis secundaria) posiblemente Leuconostoc sp era parte de la flora polimicrobiana, resultando pertinente considerar su participación en el proceso infeccioso y, por lo tanto, en la elección del tratamiento antimicrobiano más apropiado, como apoyo a la remoción mecánica del foco infeccioso.

Un antecedente importante en todos los pacientes fue el uso previo de antibacterianos de amplio espectro (cefepime, piperacilina/tazobactam, meropenem, clindamicina y amikacina), antimicrobianos que fueron indicados para los episodios de neutropenia y fiebre post-quimioterapia, en los cuales es recomendado utilizar antimicrobianos con cobertura para Pseudomonas aeruginosa y en algunas ocasiones, según los criterios clínicos, vancomicina empíricamente para cubrir cocáceas grampositivas resistentes a meticilina. Seis de los nueve pacientes habían recibido, en algún momento del tratamiento para las complicaciones infecciosas relacionadas con cáncer, vancomicina. Sólo uno de los nueve aislados de Leuconostoc sp mostró un patrón de sensibilidad intermedia a penicilina. Siete cepas de Leuconostoc fueron, además, sensibles a oxacilina, rifampicina, cotrimoxazol y ciprofloxacina; una de las cepas fue resistente a todos ellos. Los nueve aislados fueron resistentes a vancomicina.

La muestra clínica de la cual se recuperó Leuconostoc sp con mayor frecuencia fue la del torrente sanguíneo, en cuatro oportunidades.

Dos de los nueve pacientes estaban hospitalizados en la Unidad de Cuidados Intensivos en el momento de la obtención del cultivo. Tres pacientes fallecieron por causas que no se pueden atribuir directamente al aislado.

\section{Discusión}

Las especies de Leuconostoc no son parte de la microbiota en los seres humanos; a pesar de ello, de forma ocasional se ha aislado en secreciones vaginales y en deposiciones de personas sanas. Igualmente, de forma poco común, se han hecho descripciones de infección por este microorganismo en sangre, CVC empleado en nutrición parenteral, LCR, orina y hueso, especialmente en pacientes inmunocomprometi$\operatorname{dos}^{2-4,14-17}$.

Aunque la población afectada puede ser de cualquier edad, predomina en niños y ancianos. Se cree que el medio de acceso del microorganismo al cuerpo es a través de la pérdida de la integridad de las barreras muco-cutáneas, piel y mucosa gastrointestinal, aunque no se conoce aún el mecanismo de transmisión ni los posibles reservorios. Algunos reportes muestran el aislado de Leuconostoc sp en pacientes con nutrición enteral o parenteral ${ }^{14}$. El cuadro clínico más frecuentemente documentado es el de bacteriemia asociada a CVC, aunque en la literatura médica se encuentran reportes de meningitis, abscesos mamarios, infecciones de vías urinarias, peritonitis y colecciones intraabdominales $^{5,6,16}$.

Desde su primera descripción en 1985, se han identificado poblaciones susceptibles asociadas con los extremos de la vida, siendo los niños los más afectados y factores condicionantes la premature ${ }^{14}$, las anormalidades congénitas del tracto gastrointestinal y las quemaduras graves. Otros factores de riesgo identificados son la presencia de dispositivos tales como cánula de traqueostomia, gastrostomia, o tubos oro-traqueales $^{7-9}$.

Dentro de las condiciones clínicas que con mayor frecuencia se han documentado en dichos pacientes se mencionan la diabetes mellitus, insuficiencia renal crónica, infección por VIH y SIDA, artritis reumatoidea, hepatopatía alcohólica y antecedente de cáncer. Igualmente, el uso prolongado de antimicrobianos de amplio espectro que incluyen cotrimoxazol, $\beta$-lactámicos, aminoglucósidos y vancomicina; también se ha relacionado con la selección de especies intrínsecamente resistentes a glucopéptidos ${ }^{10,11}$.

El uso de vancomicina en la mayoría de los pacientes descritos aquí, probablemente, también es un factor condicionante que permitió la selección de la microbiota y de cocáceas grampositivas con resistencia primaria a glucopéptidos, como es el caso de Leuconostoc sp. Esta bacteria posee una enzima penta depsipéptido ligasa $\mathrm{LmDd}^{12}$, con un carbono C terminal D-lactato en vez de D-alanina, lo que genera un péptido intermediario terminado en D-alanina D-lactato, con menor afinidad por los glucopéptidos ${ }^{14}$.

Ésta es la primera serie latinoamericana de casos descritos en pacientes inmunocomprometidos como factor de riesgo para el desarrollo de infecciones por Leuconostoc sp. Incluso en pacientes con neutropenia y fiebre, su papel patogénico no es claro, teniendo en cuenta que su presencia no se asoció directamente con mortalidad. Debido a que el uso de vancomicina ${ }^{18}$ se recomienda en bacteriemias por cocáceas grampositivas, los laboratorios de los centros oncológicos deben estar capacitados para detectar las especies de Leuconostoc sp con el fin de evitar el sobreuso de vancomicina.

En esta serie, la principal co-morbilidad identificada fue la presencia de neoplasia hematológica y los episodios de neutropenia y fiebre post-quimioterapia y de neoplasia de órgano sólido en estadios avanzados de enfermedad y en progresión. Los factores condicionantes que facilitaron la presencia de Leuconostoc 
sp en estos pacientes, probablemente obedecen a compromiso de la inmunidad innata por la enfermedad de base y por el uso de la quimioterapia; compromiso de la inmunidad celular debido a la presencia de neoplasias de órgano sólido que se manifiesta con un alto nivel de catabolismo, desnutrición proteico-calórica, y la utilización de dispositivos intravasculares necesarios para el tratamiento de apoyo que estos pacientes requieren, por lo que, en este grupo de pacientes, Leuconostoc sp se puede considerar como un patógeno oportunista.

Debido a que los aislados descritos en esta serie son previos al documento M45-A recomendado recientemente por la CLSI ${ }^{13}$ para las pruebas de susceptibilidad para Leuconostoc sp y otras bacterias exigentes nutricionalmente para su crecimiento in vitro, estos criterios microbiológicos no se cumplieron en los casos que aquí se informan. La mayoría de los aislados fueron sensibles a penicilina con CIM $<$ de $1 \mu \mathrm{g} / \mathrm{mL}$ al igual que a otros $\beta$-lactámicos. Aunque en la literatura científica se describe, con frecuencia, sensibilidad intermedia a penicilina, esta observación fue la excepción en nuestra experiencia y la resistencia es menos frecuente si se considera que sólo se identificó una cepa multi-resistente.

Para el tratamiento de este microorganismo se pueden emplear varios antimicrobianos, entre los que se cuentan: $\beta$-lactámicos como penicilina y ampicilina, cefalosporinas y carbapenems. La penicilina y ampicilina se consideran los antimicrobianos de primera elección, así como los macrólidos en pacientes con antecedente de alergia a penicilina. Otros antibacterianos como los aminoglucósidos y clindamicina también pueden ser útiles en el tratamiento.

El pronóstico de la infección y la respuesta terapéutica dependen del sistema inmune del hospedero y de identificar los focos primarios de infección para que, en los casos que sea procedente, se retiren los cuerpos extraños (dispositivos intravasculares) o se drenen las colecciones ${ }^{1,3,4,12}$.

\section{Conclusiones}

En los pacientes que presentan condiciones clínicas y que además necesitan del uso de dispositivos intravasculares, nutrición parenteral, tratamiento con vancomicina y que cursen con patologías que comprometan su inmunidad innata y adquirida, (nueve pacientes esta serie de casos), tienen alto riesgo para desarrollar infecciones oportunistas por Leuconostoc sp; esta cocácea grampositiva se debe considerar en el análisis de las posibles causas infecciosas en pacientes con cáncer que no responden al uso de glucopéptidos, por ser intrínsecamente resistentes a los mismos.

En la revisión de la literatura médica efectuada por los autores, éste es el primer informe de pacientes en Latinoamérica con patología oncológica en quienes se aísla Leuconostoc sp como patógeno oportunista. Su papel en la mortalidad y la posibilidad de transmisión de la resistencia no son claros, pero su aparición llama al uso juicioso de vancomicina en pacientes con cáncer.

\section{Resumen}

Las especies de Leuconostoc son patógenas oportunistas informadas en muy baja frecuencia, que se pueden encontrar en pacientes críticamente enfermos, inmunocomprometidos y con infecciones intrahospitalarias. Generalmente, se asocian a bacteriemia por dispositivos intra-vasculares y al uso de nutrición parenteral total. Sin embargo, también se han descrito otras infecciones asociadas, dentro de las que se cuentan meningitis, osteomielitis, infección del torrente sanguíneo, de vías urinarias y peritonitis. En este artículo se describe una serie de pacientes con cáncer en quienes se identificó este microorganismo, las condiciones clínicas asociadas y se hace una revisión de la literatura.

\section{Referencias}

1.- Dhodapkar K M, Henry N K. Leuconostoc bacteriemia in an infant with short-gut syndrome: case report and literature review. Mayo Clin Proc 1996; 71: 1171-4.

2.- Espinoza R, Kusne S, Pasculle A W, Wada S, Fung J, Rakela J. Leuconostoc bacteremia after liver transplantation: another cause of vancomycin resistant gram-positive infection. Clin Transp 1997; 11: 322-4.
3.- Goenaga S. Bacteremia por Leuconostoc spp en un paciente con síndrome de pseudoobstrucción intestinal. Anales Medicina Interna 2003; 20: 61 .

4.- Mulford J S, Mills J. Osteomyelitis caused by Leuconostoc species. Aust N Z J Surg 1999; 69: 541-2.

5.- Scano F, Rossi L, Cattelan A, Carretta G, Meneghetti F Cadrobi P, et al. Leuconostoc species: a case-cluster hospital infection. Scand J Infect Dis 1999; 31: 371-3.
6.- Friedland I, Snipelisky M, Manikant K. Meningitis in a neonate caused by Leuconostoc sp. J Clin Microbiol 1990; 29: 2125-6.

7.- Jato A, García-Zarbate M, Jato M, Varela E, García-Riestra C, Regueiro B. Bacteriemia en una niña con rabdomiosarcoma. Enferm Infecc Microbiol Clin 2002; 20: 85-7.

8. - Taneja N, Rani P, Emmanuel R, Khudaier B Y, Sharma S K, et al. Nosocomial urinary tract infection due to Leuconostoc mesenteroides at a tertiary 
care centre in North India. Indian J Med Res 2005; 122: 178-9.

9.- Gillespie R, Symons J, Mcdonald R. Peritonitis due to Leuconostoc species in a child receiving peritoneal dialysis. Pediatr Nephrol 2002; 17: 966-8.

10.- Jiménez-Mejías M E, Becerril B, GómezCía T, Del Nozal M, Palomino-Nicán J. Bacteremia caused by Leuconostoc cremoris in a patient with severe burn injuries. Eur J Clin Microbiol Infect Dis 1997; 16: 533-4.

11.- Vagiakou-Voudris E, Mylona-Petropoulou D, Kalogeropoulou E, Chantzis A, Chini S, Tsiodra P, et al. Multiple liver abscesses associated with bacteremia due to Leuconostoc lactis. Scand J Infect Dis 2002; 34: 766-7.

12.- Kulwichit W, Nilgate S, Krajiw S, Srisawat N,
Unhasuta C, Chongthaleong A. Enigmatic Leuconostoc and Lactobacillus infections: P1720. Clin Microb Infect 2005; 11 (Suppl 2): 564.

13.- Jorgensen J H, Hindler J F. New consensus guidelines from the Clinical and Laboratory Standards Institute for antimicrobial susceptibility testing of infrequently isolated fastidious bacteria. Clin Infect Dis 2007: 44: 280-7.

14.- Jofré L, Sakurada A, Ulloa T, Hormazábal J C, Godoy V, Fernández J, et al. Infección por Leuconostoc en pacientes con síndrome de intestino corto, nutrición parenteral y alimentación enteral contínua. Rev Chil Infect 2006; 23: 340-5.

15.- Cappelli E, Barros R, Camello Th,
Teixeira L, Merquior V L. Leuconostoc pseudomesenteroides as a cause of nosocomial urinary tract infections. J Clin Microbiol 1999; 37: 4124-6.

16.- Zaoui A, Brousse Ch, Bletry O, Augouard L W, Boisaubert B. Leuconostoc osteomyelitis. Joint Bone Spine 2005; 72: 79-81.

17. - Fauchais A L, Roques S, Druesne L, Verdonck A, Chassagne P, Bercoff E, et al. Rare opportunistic infection due to Leuconostoc. Rev Med Interne 2003; 24: 268-75.

18.- Hughes W T, Armstrong D, Bodey G P, Bow E J, Brown A E, Calandra T, et al. 2002 Guidelines for the use of antimicrobial agents in neutropenic patients whit cancer. Clin Infect Dis 2002; 34: 730-48. 